\title{
The Case for Oxygen in Global Surgical Care
}

\section{K. A. Kelly McQueen ${ }^{1}$}

Published online: 20 April 2016

(c) Société Internationale de Chirurgie 2016

\section{Dr.'s Flescher and Patel}

Thank you for your recent letter to editor further highlighting the critical need for continuous oxygen availability in low-income countries where surgery is provided. You make the important point for pre-oxygenation prior to emergency airway management, and this is, of course, important not only in the surgical theater, but also in the emergency department, the obstetrical delivery suites, and on pediatric and other high dependency units.

As hospitals in low-income countries scale up to treat non-communicable disease, including trauma and many other surgical diseases, the role of oxygen must be carefully planned for, and as you point out, an early investment in a reliable, continuous oxygen supply and related delivery supplied is essential.
K. A. Kelly McQueen

kelly.mcqueen@vanderbilt.edu

1 Departments of Anesthesiology and Surgery, Vanderbilt Institute for Global Health, Vanderbilt University Medical Center, 1301 Medical Center Drive, MCE 3161C, Nashville, TN 37232, USA 